\title{
Microscopic agents programmed by DNA circuits.
}

\author{
G. Gines ${ }^{1}$, A.S. Zadorin ${ }^{2,3}$, J.-C Galas ${ }^{2}$, T. Fujii ${ }^{1}$, A. Estevez-Torres ${ }^{2}$, Y. Rondelez ${ }^{1,3^{* *}}$ \\ 1. LIMMS, CNRS/Institute of Industrial Science, UMI 2820, University of Tokyo, 153-8505 Tokyo, Japan. 2. Laboratoire Jean Perrin, \\ CNRS/Université Pierre et Marie Curie, UMR 8237, 4 place Jussieu, 75005 Paris, France. 3. Laboratoire Gulliver, CNRS/Ecole Supérieure de \\ Physique et Chimie Industrielles, UMR 7083, 10 rue Vauquelin, 75005 Paris, France. \\ **email : yannick.rondelez@espci.fr
}

Information stored in the sequence of synthetic nucleic acids can be used in vitro to create complex reaction networks implementing precisely programmed chemical dynamics. We report here the extension of this approach to program the local and individual chemical behaviour of microscopic "agents" dispersed in an enzymatic solution. Agents possess multiple stable states, thus maintaining a memory, and communicate by emitting various orthogonal signals and sensing the behaviour of neighboring agents. We build on these elements to create collective behaviours involving thousands of different agents, for example retrieving information over long distances, or creating spatial patterns. This approach recapitulates some fundamental mechanisms of distributed decision making and morphogenesis among living organisms. The possibility to scale up the information-processing capability of DNA-encoded artificial systems could also find applications where many individual clues need to be combined to reach a decision, e.g. in molecular diagnostics.

In the last decade, the emergent field of molecular programming has crafted an array of molecular systems that perform complex information-processing functions using networks of biomolecular components ${ }^{1,2}$. Many of these man-made systems are inspired by regulatory networks observed within single cells: logic circuits seen in signaling cascades $^{3,4}$; circadian oscillators pacing the metabolism of light-harvesting microorganisms ${ }^{5,6}$; all-ornothing switches embedded in genetic regulation pathways ${ }^{4,7,8}$, shape-forming morphogen fronts in developing embryos ${ }^{9-12}$. These systems are based on homogeneous mixtures where a unique computation happens in one bulk solution. In contrast to these amorphous systems, a number of interesting biological behaviours result from the cooperation of many physically separated units exchanging molecular information, through diffusion for example. Bacteria use quorum sensing to collectively select survival strategies1 ${ }^{13,14}$, multiple cells within embryos exchange morphogens to generate sharp differentiated clusters ${ }^{15,16}$, and unicellular organisms such as Dictyostelium switch to a multicellular lifestyle to overcome adverse conditions ${ }^{17}$.

Inspired by these forms of distributed intelligence, we set out to fabricate arrays of particles that are programmed at the molecular scale but perform collective behaviours encompassing many objects. We first adapt molecular programming techniques to a surface-supported format. To create particles that communicate selectively with each other we build here on the Polymerase/Exonuclease/Nickase (PEN) Dynamic Network Assembly toolbox 5,18, a molecular programming scheme where short oligodeoxyribonucleotides (templates) encode the connectivity information of a network of activating/inhibiting interactions (Fig. 1a). We attach these rule-encoding DNA templates to micrometric porous particles (Fig. 1b). The particles are then immersed within a solution containing the PEN enzymes, leading to the interpretation of the particle-bound DNA rule into a local chemical behaviour (Fig. 1c-d). We show that the individual behaviour of a bead carrying a tethered PEN DNA network qualitatively reproduces the one observed for solution-phase systems with the same set of templates. However, production of DNA signals is now spatially constrained to the position of the bead, while degradation extends to the bulk of the solution, resulting in the generation of concentration gradients around each agent (Supplementary Notes 1). This creates autonomous, mono- or multi-stable particles that communicate locally through diffusive exchange of signals (Fig. 1e).

These building blocks enable the rational molecular programming of population-scale phenomena ${ }^{19,20}$. We generate spatially random arrays containing thousands of particles in which we observed the propagation of bistable travelling fronts, or the generation of stationary symbiotic activation patterns, depending on the molecular instructions carried by the microscopic agents. We also show that multiple orthogonal layers of chemical communication can be used in a single system. We used this capability to build an assembly where discrete agents cooperate to propagate chemical information over a distance of a few millimeters, check for the presence of a particular bead type, and carry this information back to the origin of the query (Fig. 1e, right). 

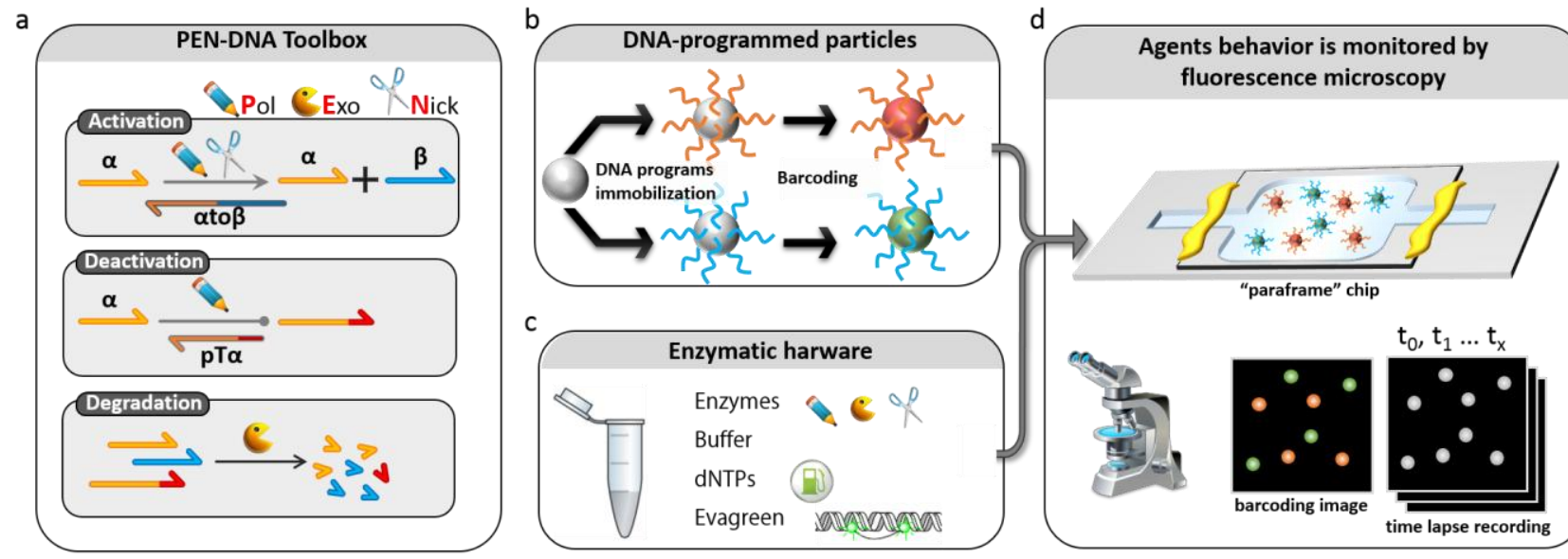

"paraframe" chip
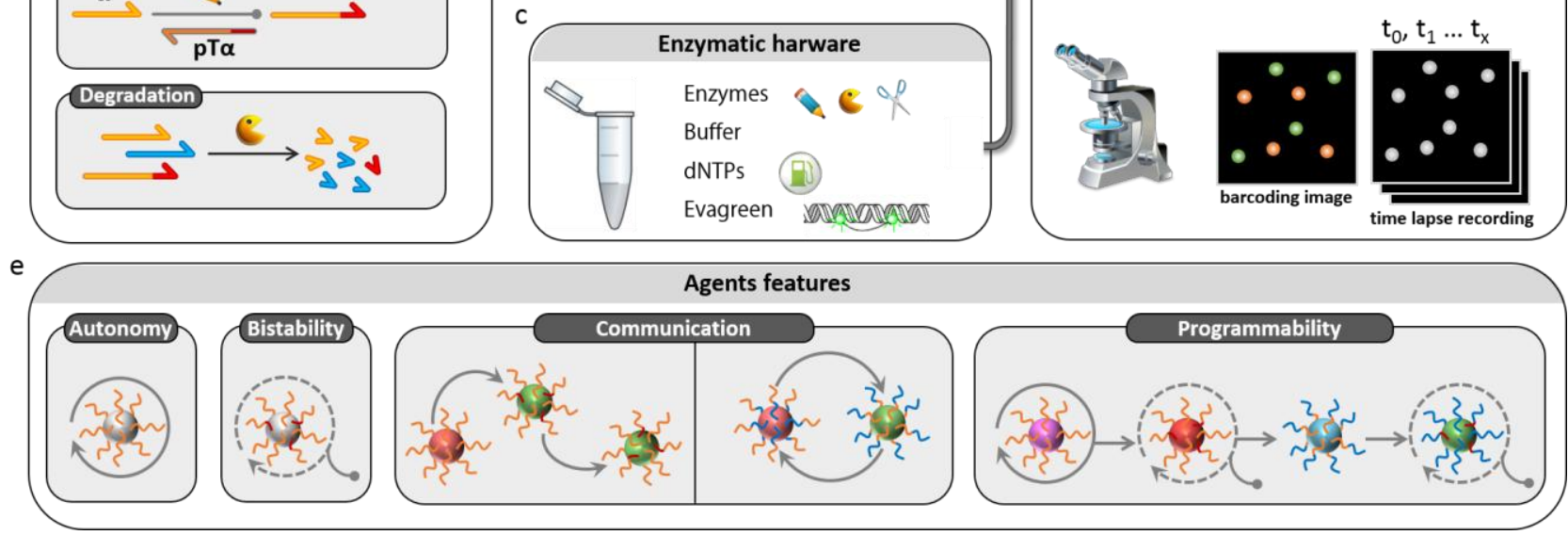

Figure 1. Programming microscopic agents using DNA-encoded reaction rules. a, The PEN DNA toolbox is a set of generic modules that can be cascaded to build molecular programs processed by an enzymatic machinery. $\mathbf{b}$, In this study, we convert the solution-phase system to a spatially discrete architecture by attaching the rule-encoding templates to micrometric particles. Particles are barcoded to be easily distinguished by fluorescence according to the set of templates they carry. c, The reaction mix contains the enzymatic hardware in addition to the buffer, the fuel dNTPs and a double strand-specific dye. d, Particles are incubated in a 2-dimensionnal micro-chamber and the reaction (creation of output) is monitored by microscopic imaging of the EvaGreen fluorescence signals, reflecting the relative amount of active (i.e. double-stranded) templates on each particle. In the case of mixed populations, barcode channels are used to identify the circuit carried by each particle. e, Programmed particles behave as independant microscopic agents displaying features such as bistability, communication and programmability.

\section{Particles encoded with a positive feedback loop}

The dynamics of PEN DNA systems, relies on a generalist enzymatic machinery that drives the exchange of DNA signals between the rule templates: a DNApolymerase elongates an input strand that hybridizes on the input (3') side of a matching template; a nickase recognizes and cuts the resulting full duplex, releasing both the input and a new output DNA. The exonuclease non-specifically degrades all unprotected oligonucleotides (templates are protected), maintaining the system in a responsive out-of-equilibrium state. In functional terms, hybridization and enzymatic reactions collaborate to generate a set of basic modules that can be cascaded in dynamic circuits whose parameters are linked to kinetic, thermodynamic and concentration values. This versatile system has already allowed the construction of various solution-phase circuits such as oscillators ${ }^{5}$ or multistable switches ${ }^{8}$.

We first investigated the possibility of grafting ruleencoding DNA templates onto solid supports to create spatially localized DNA programs ${ }^{21,22}$ (Supplementary
Methods; all sequences are provided in Supplementary Table 1). Streptavidin-conjugated porous microparticles (diameter $\varnothing=34 \pm 10 \mu \mathrm{m}$ ) were functionalized to the biotin-modified oligonucleotide ato $\alpha$, a dual-repeat sequence catalysing the positive feedback loop (PFL) reaction $\alpha \rightarrow 2 \alpha$ (Fig. 2a). At sub-saturating grafting levels, stable, shell-like objects are obtained (Supplementary Figs 1-4). These PFL-encoded particles are then transferred to a master mix containing PEN enzymes and incubated in a flat microscopy chamber. The amplification reaction is monitored using the double strand binding dye EvaGreen that fluoresces on binding the active templates producing the $\alpha$ strand (Supplementary Fig. 5a). Time-lapse fluorescence imaging revealed the expected behaviour for the PFL circuit: an initial exponential amplification phase is followed by a steady-state plateau (production balances diffusion and degradation), then a return to the baseline after exhaustion of dNTPs (Supplementary Fig. 5 and Supplementary Movie 1). This behaviour closely parallels that observed for the case of freely diffusing 
templates in bulk reactions. In the case of particletethered templates, however, strong changes in fluorescence signal are observed only within the particles and not in the bulk of the solution. Therefore, the production of output $\alpha$ is now localized, and its degradation occurs in the rest of solution.

The dimensionless Damköhler number Da, defined as the ratio of the chemical reaction rate to the diffusive transport rate, gives an estimate of the importance of local versus global effects in chemical systems (for details of the estimation of $\mathrm{Da}$ and reaction- diffusion length scales, see Supplementary Note 1). Here, the Da associated to grafted particles is much greater than 1 , so we expect a dominance of local effects. Ultimately, a single particle performing an autocatalytic reaction should be able to sustain, on its own, a positive feedback loop, despite being constantly depleted by diffusion. To check this self-sustained reactivity, a unique particle was incubated in a large chamber (volume ratio of $10^{-6}$, Fig. $2 b)$. Here, the total amount of template would be too small to sustain amplification in a bulk solution $(\sim 3 \mathrm{fmol}$ for a total volume of $10 \mu \mathrm{l}$, that is, less than one nanomolar). The strands are, however, highly concentrated within the particle, and we did observe a sharp localized amplification that was maintained autonomously for more than $18 \mathrm{~h}$. The production is strictly constrained on the particles, but the signal strands are free to diffuse, leading to a concentration gradient around the autocatalytic particle, which we term a 'diffusion cone'. We analytically solved the steady-state concentration profile generated by shell-like selfactivating particles in the presence of a global sink (Supplementary Note 2). Above a critical size, or grafting density, a stable concentration profile is generated, the precise mathematical expression of which will depend on the dimensionality of the problem ${ }^{23}$, but whose length parameter is always controlled by $\sqrt{D / k}$ (where $\mathrm{D}$ is the diffusion rate and $\mathrm{k}$ the first-order degradation rate for the signaling compound). Under our conditions, these parameters are $\mathrm{D}=18 \times 10^{3} \mu \mathrm{m}^{2} \min ^{-1}$ and $\mathrm{k}=0.07$ $\min ^{-1}$ (Supplementary Note 3 ), suggesting that active particles generate a steady-state diffusion 'cone' with a length scale of $\sim 500 \mu \mathrm{m}$. To observe these profiles directly, we added a reporter template $\alpha$ to $\beta$ into the experimental system. $\alpha$ to $\beta$ binds the diffusing signal $\alpha$ and, upon extension and nicking, produces a futile $\beta$ strand. This process involves transient generation of double-stranded DNA and, in the presence of EvaGreen, reversibly reveals the presence of $\alpha$. The template $\alpha$ to $\beta$ is attached to specialized reporter beads randomly distributed in the chamber, so that the reporting chemistry does not introduce additional diffusion effects (Fig. 2c). This experimental approach exposes cone-shaped fluorescence profiles surrounding active autocatalytic particles (Fig. 2d). With an exonuclease acting as a degradation mechanism in the solution, the profiles stabilize after $\sim 10 \mathrm{~h}$ (Fig. 2d, left and Supplementary Movie 2). Numerical fits to a modified Bessel function of the second kind (see Supplementary Note 3 for details) allow us to extract the characteristic length $l_{\mathrm{d}}$ of the diffusion cones, with a steady-state value of $400 \pm 100$ $\mu \mathrm{m}$, in good agreement with predictions. When the exonuclease is absent, fluorescence gradually increases everywhere in the chamber and we do not observe a steady state (Fig. 2d, right). We noticed that, despite the absence of initial trigger in the chamber, particles carrying the template $\alpha$ to $\alpha$ inevitably switch to the "ON" (productive) state after a short period of incubation (Fig. 2b). This phenomenon is again consistent with the behaviour reported for solution-phase amplification systems: autocatalytic templates self-start after some time because the first-order PFL network enforced by the dual repeat template is intrinsically monostable. ${ }^{24}$ In order to stabilize the "OFF" state (corresponding to a nonproductive state) we use a species-specific deactivation template (pseudo-template, pT) that catalyzes the addition of a short 3' tail to the trigger of the associated template (Fig. 2e and Fig. S6). Like the autocatalytic template the pseudo-template is composed of a 3' input binding part, but its output part consists of only a few nucleotides (poly-dA or poly-dT). As the trigger binds to the $\mathrm{pT}$, it is elongated by the polymerase. The resulting extended strand slowly melts away (regenerating the pseudo-template so that this mechanism is catalytic) and is now unable to prime further reactions. This mechanism constitutes a signal-specific sink of tunable throughput that has been used to build multistable and excitable circuits ${ }^{25}$. We adapt this strategy to particle systems by co-immobilizing the autocatalytic template with the corresponding pseudo-template on the particles. Microscopy experiments (Fig. 2f) show that co-grafted particles now possess two different stable states (nonproductive and productive, referred as "OFF" and "ON" states), which are selected depending on the initial conditions (respectively high or low trigger concentration). Pseudo-templates are therefore able to abolish self-start, even in the context of the very high local concentration of templates $(\sim 250 \mu \mathrm{M}$ in the bulk of the sphere). If no signal is present in their environment the particles stay in their OFF state, so that the activation of bistable particles necessarily relies on the application of a stimulus exceeding a given threshold. In the following, we call the particles carrying only the autocatalytic template $\mathrm{P} \alpha_{\mathrm{M}}$ and the co-grafted particles $\mathrm{P} \alpha_{\mathrm{B}}$ ( $\mathrm{M}$ and $\mathrm{B}$ stand for monostable and bistable, respectively). 


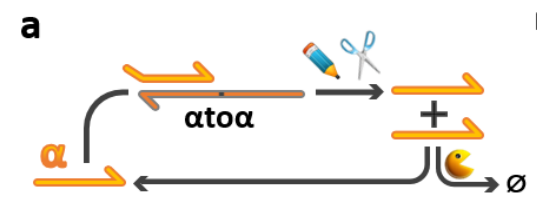

Monostable $\left(P \alpha_{M}\right)$

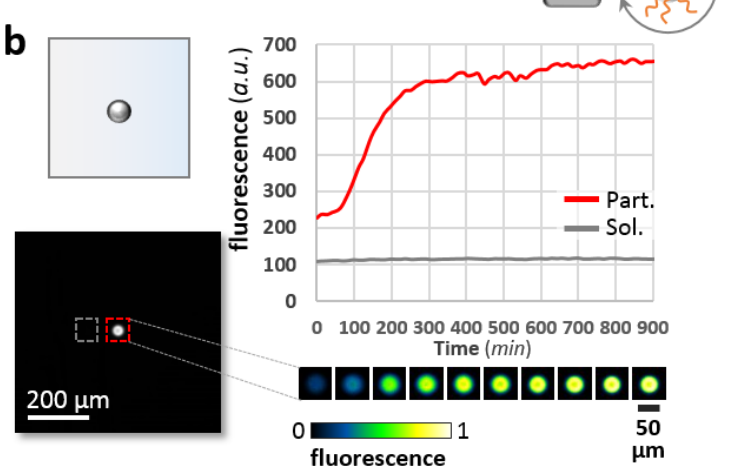

c

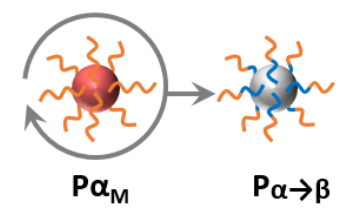

d

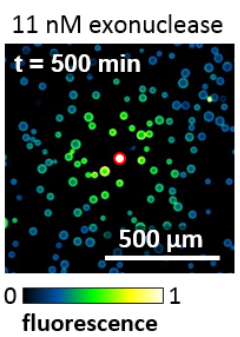

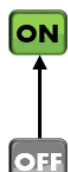

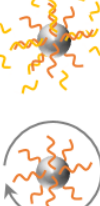

e

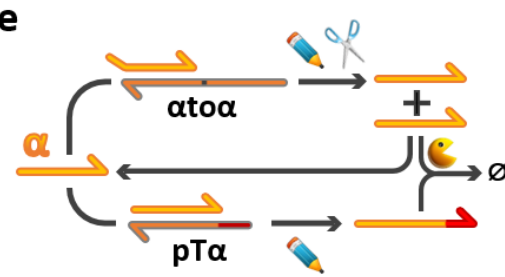

Bistable $\left(P \alpha_{B}\right)$

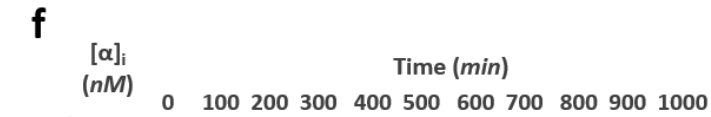

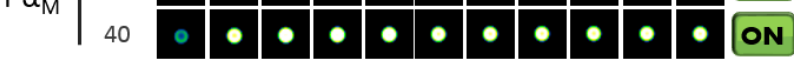

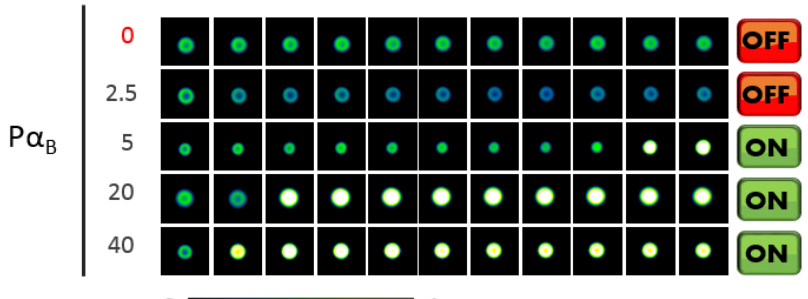

fluorescence
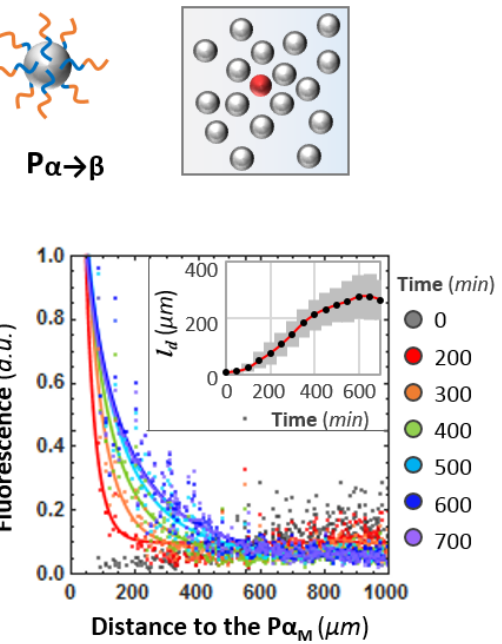
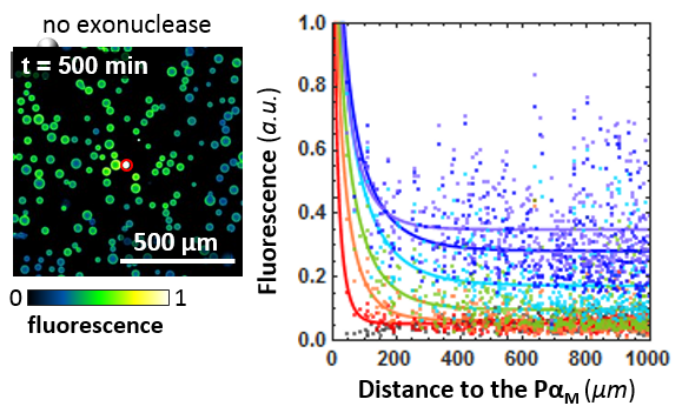

Figure 2. Positive feedback loop-encoded agents. a, A PFL encoded by the template $\alpha$ to $\alpha$ catalyzes the self-activated production of $\alpha$ strands. The $\alpha$ to $\alpha$ template is grafted to porous microparticle to obtain intrinsically monostable agents $\mathrm{P} \alpha \mathrm{M}$ that spontaneously switch to the high state. $\mathbf{b}$, Microscopic fluorescence recording of the amplification reaction on a single particle in a flat micro-chamber (particle to chamber volume ratio is $1: 10^{6}$ ) in absence of initial trigger $\alpha$. The amplification reaction is monitored with a double strand specific dye that fluoresces upon binding to the (doublestranded) active template. c, Schematic of the network design to assay the diffusion cone generated by autocatalytic PFL-encoded agents: $\mathrm{P} \alpha \mathrm{M}$ is surrounded by reporter particles $\left(\mathrm{P}_{\alpha_{\rightarrow} \beta}\right)$ functionalized by the template $\alpha$ to $\beta$. Upon the capture of $\alpha$ strands by $\mathrm{P}_{\alpha \rightarrow \beta}$, the latter produces a futile $\beta$ strand. The transient formation of double-stranded $\alpha$ to $\beta$ templates increases fluorescence and reveals the presence of $\alpha$. d, Cone-shaped fluorescence profiles surrounding P $\alpha \mathrm{M}$ in presence (left) or in absence (right) of a degradation sink (exonuclease). Microscopy images are extracted at $\mathrm{t}=500$ min. The fluorescence of the reporter particles (extracted after segmentation) is plotted for different times of the reaction as a function of their distance to the $\mathrm{P} \alpha \mathrm{M}$. The solid lines represent numerical fits to a modified Bessel function of the second kind $f_{2 D}(r)=a K_{0}\left(r / l_{d}\right)+b$, where $r$ is the distance to the autocatalytic particle, $l_{d}$ represents the characteristic length of the diffusion cone, $a$ and $b$ are constants. The inset shows the evolution to the steady-state of $l_{d}$ over time. e, Bistable design based on a fast but saturable deactivation pathway targeting the autocatalytic species $\alpha$. The two templates ( $\alpha$ to $\alpha$ and $\mathrm{pT} \alpha)$ are co-grafted on beads to obtain bistable agents $\left(\mathrm{P} \alpha_{\mathrm{B}}\right)$ that require specific triggering to switch on. f, Panel of snapshots extracted from time-lapse fluorescence recording of individual particles $P \alpha_{M}$ or $P \alpha_{B}$ incubated with various initial amounts of $\alpha$ strand $(0 \leq[\alpha] \leq 40 \mathrm{nM})$. 
a

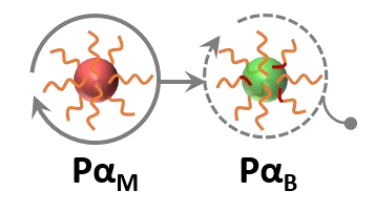

self-

ignition Traveling front propagation

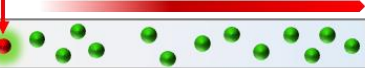

d

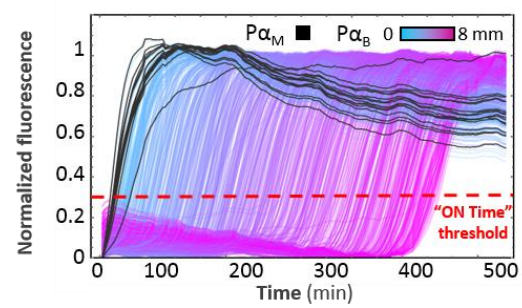

b

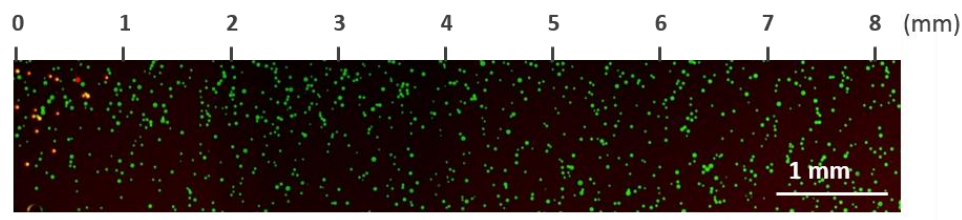

C

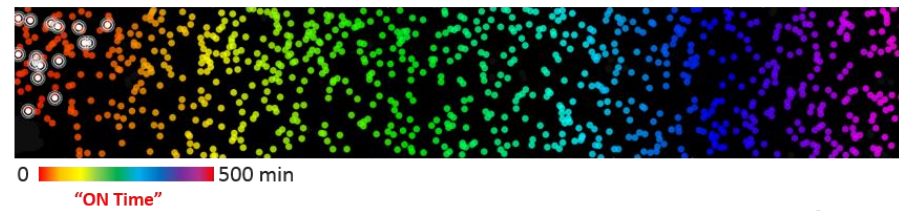

e
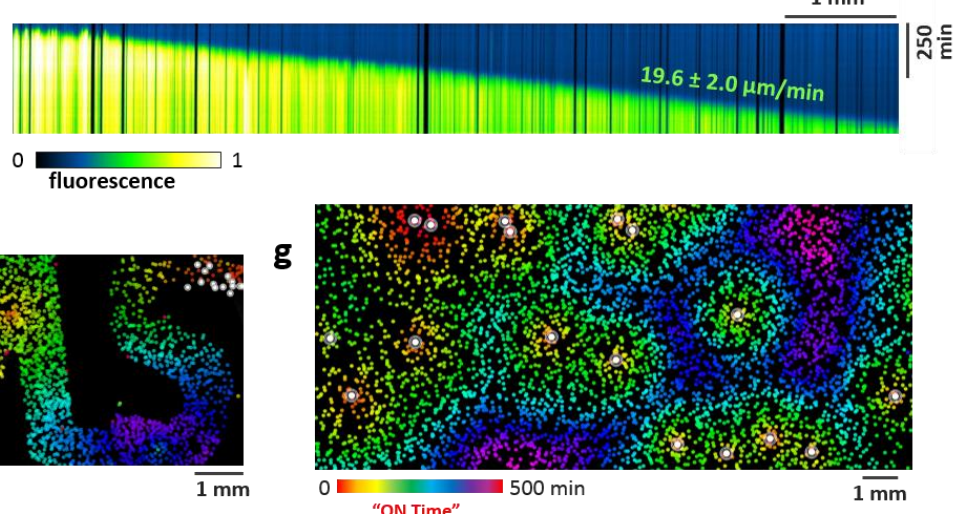

"ON Time"

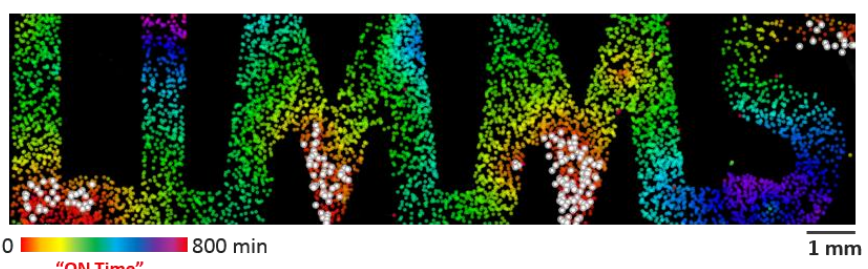

Figure 3. Travelling front propagation across a population of bistable agents $\left(\mathbf{P} \boldsymbol{\alpha}_{\mathbf{B}}\right)$. $\mathbf{a}$, Schematic representation showing the propagation of an activation signal triggered initially by the self-ignition of monostable agents $\left(\mathrm{P} \alpha_{\mathrm{M}}\right)$. $\mathbf{b}$, Barcode image (red dots $=\mathrm{P} \alpha_{\mathrm{M}}$; green dots $=\mathrm{P} \alpha_{\mathrm{B}}$ ). c, Colorized representation of the front propagation where color represents the time of amplification ("ON" time) for each particle. The white spots indicate the positions of the P $\alpha_{M}$. d, Fluorescence time traces extracted for each particle show the spontaneous switching of monostable particles and the correlation between the position of bistable particles and their amplification start time. e, kymogram revealing the constant velocity ( $\mathrm{v}$ ) of the travelling front across the population of $\mathrm{P} \alpha_{\mathrm{B}}$. f,g, Traveling front propagation across populations of $P \alpha_{B}$ in various geometric environments and dispositions of the $P \alpha_{M}$ (marked by white spots) in the chamber. For details on image processing see Fig. S11.

\section{Travelling front propagation in a population of bistable agents}

Figure $3 \mathrm{a}$ depicts an experiment where bistable particles $\mathrm{P} \alpha_{\mathrm{B}}$, initially in their OFF state, are randomly positioned in an extended channel roughly one centimeter long. On one end of the channel, dye-barcoded monostable particles $\mathrm{P} \alpha_{\mathrm{M}}$ (without pseudo-template) are introduced (Fig. 3b). As expected, $\mathrm{P} \alpha_{M}$ particles self-activate after a short delay. The concentration inhomogeneity that they create is strong enough to trigger the switching of neighboring dormant $\mathrm{P} \alpha_{\mathrm{B}}$, which in turn lights up and propagates the signal to other bistable particles (Fig. 3c-d and Supplementary Movie M3). This generates a travelling front that gradually converts all particles in the channel. Switch-on times for $\mathrm{P} \alpha_{\mathrm{B}}$ particles correlate linearly to the distance from the monostable triggering beads (Fig. 4e).

The front propagates more slowly than if templates where free in solution (Fig S7), and its velocity can be finely tuned between a few to tens of micrometers per minute by tinkering with the agents' features (ratio template/pseudo-template, Fig. S8) or the experimental conditions (such as enzymes concentration or particle density, Fig. S9-10). Travelling of more complex fronts can be observed in environments with different geometries (Fig. 3f,g and Supplementary Movie M4).

\section{A four-agent network}

Communication between the particles need not be limited to a single molecular pathway: as observed in bacterial quorum sensing, various agents can possess distinct circuits, producing and detecting orthogonal signaling molecules ${ }^{26}$. To demonstrate this concept, we designed a network using two signaling compounds and four different kinds of agents. First, we created a second type of bistable particles producing and emitting strand $\beta$ (Fig. S12). We confirmed the orthogonality of the two pathways in travelling front experiments using a mixed population of bistable agents $\mathrm{P} \alpha_{\mathrm{B}}$ and $\mathrm{P} \beta_{\mathrm{B}} . \alpha$ and $\beta$ fronts were set to propagate in opposite directions, and affected only the corresponding particles (Fig. S13). In a second 
a

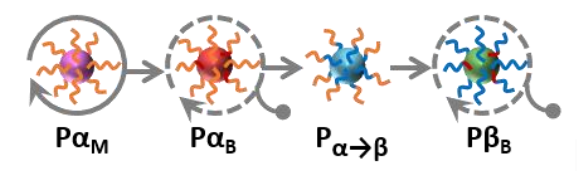

b

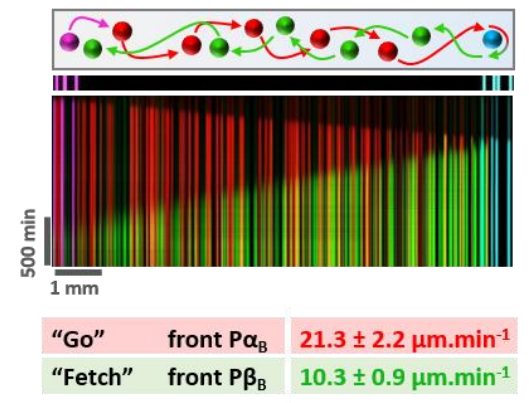

C

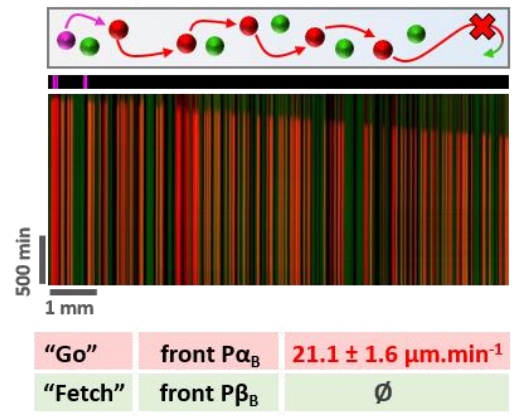

d
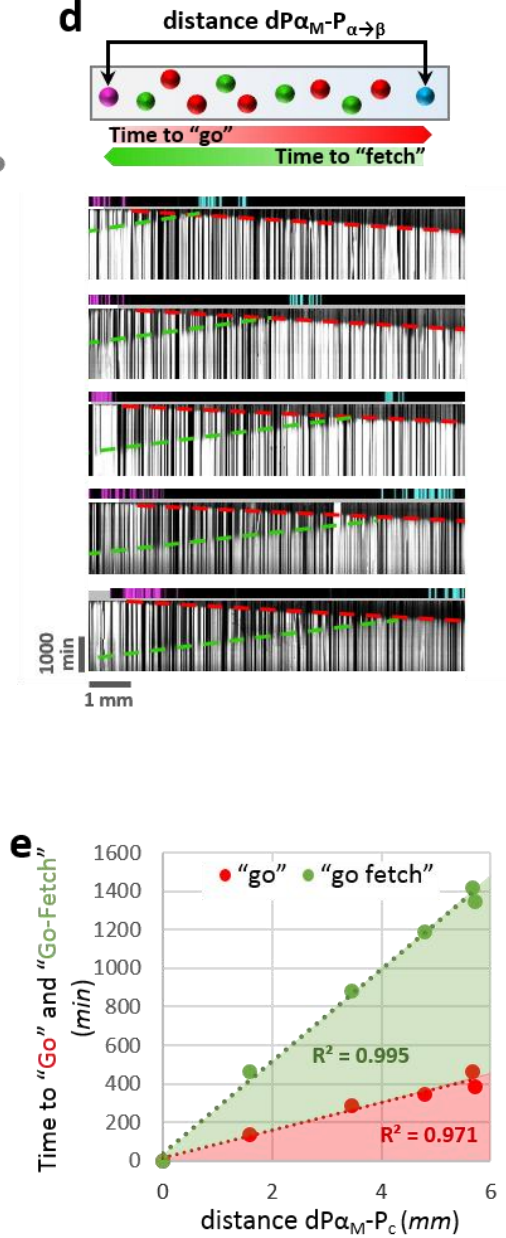

f

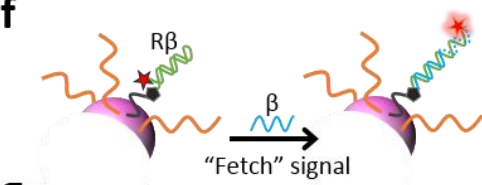

g
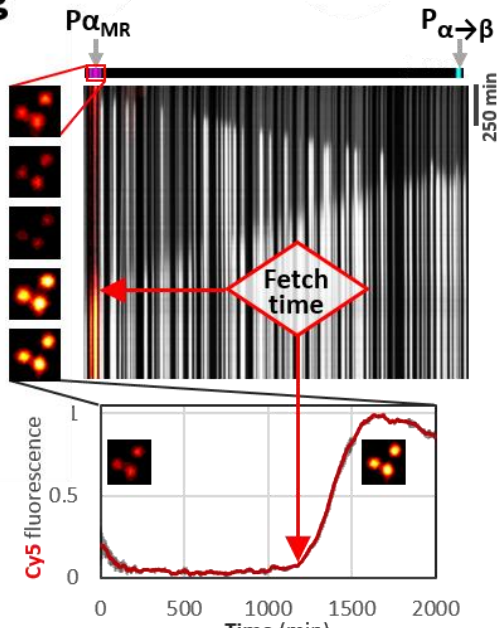

h

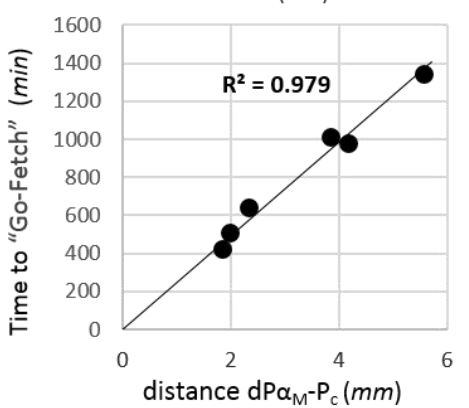

Figure 4. "Go-Fetch" program using four agent populations. a, Schematic representation showing the flow of information between the four bead types. b, Kymogram representation of the experiment. EvaGreen fluorescence signals associated with the beads are colour-coded according to the particle type (magenta $=P \alpha_{M}$, red $=P \alpha_{B}$, cyan $=$ $\mathrm{P}_{\alpha \rightarrow \beta}$, green $=\mathrm{P} \beta_{\mathrm{B}}$ ). $\mathbf{c}$, Control experiment with no converter particle at the end of the channel. $\mathbf{d}$, Converter particles are positioned at different locations in the channel. e, Front travelling time as a function of sender-target distance in d. f, An additional mechanism allows the sender particle to detect the arrival of the return front and light up. g, Experimental snapshots, kymogram and plot of the red fluorescence for sender particles. h, Light-up time of the sender particle as a function of its distance to the nearest converter.

population was coupled to the arrival of the front producing $\alpha$ using converter particles $\mathrm{P}_{\alpha \rightarrow \beta}$, carrying the template $\alpha$ to $\beta$ (which take $\alpha$ as input to produce $\beta$ as output, Fig. 4a). The topography of the spatial network, nicknamed "Go-Fetch", is as follows. Bistable agents $P \alpha_{B}$ and $P \beta_{B}$ are mixed together in a channel. At one extremity, a few monostable $\mathrm{P} \alpha_{\mathrm{M}}$ (the sender particles) are deposited; on the opposite side of the channel, the converter agents $\mathrm{P}_{\alpha \rightarrow \beta}$ represent the target. Figure $4 \mathrm{~b}$ (see also Fig. S14 and Supplementary Movie M5) shows sequentially i) the self-activation of the monostable beads $\mathrm{P} \alpha_{\mathrm{M}}$, ii) the propagation of the signal through the bistable population $\mathrm{P} \alpha_{\mathrm{B}}$, iii) the activation of the converter particles (which light on once the front reaches their position) and iv) the initiation and propagation of a second front travelling back to the initial sender, through the bistable particles $\mathrm{P} \beta_{\mathrm{B}}$.

A control experiment without converter agents also produced a first front propagation through particles $\mathrm{P} \alpha_{\mathrm{B}}$, but the return front was not generated and all particles $\mathrm{P} \beta_{\mathrm{B}}$ stayed "OFF" for the duration of the experiment (30 h). These results demonstrate the use of multiple speciated agents collaborating in a unique, spatially and time-resolved task. This program can for example be used by a sender particle to infer its distance to the nearest target particle, over a scale that is 3 orders of magnitude larger than the agents themselves. By arbitrarily varying the position of the $\mathrm{P}_{\alpha \rightarrow \beta}$ in the channel, we indeed observed that the sender particle receives this distance information as the time needed for the signal to 
return, with a linear relationship between distance and time (Fig. 4d-e). It is possible to imagine that the particles use this "chemical sonar" to control downstream processes. As a basic model of differentiation, we added a module to the sender a
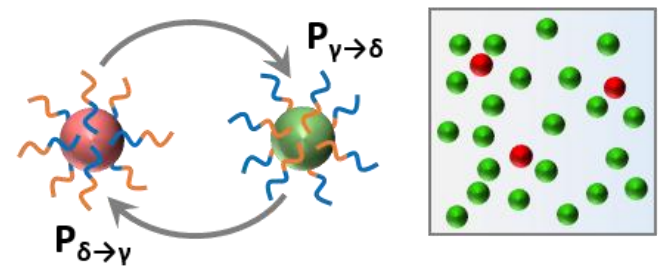

b

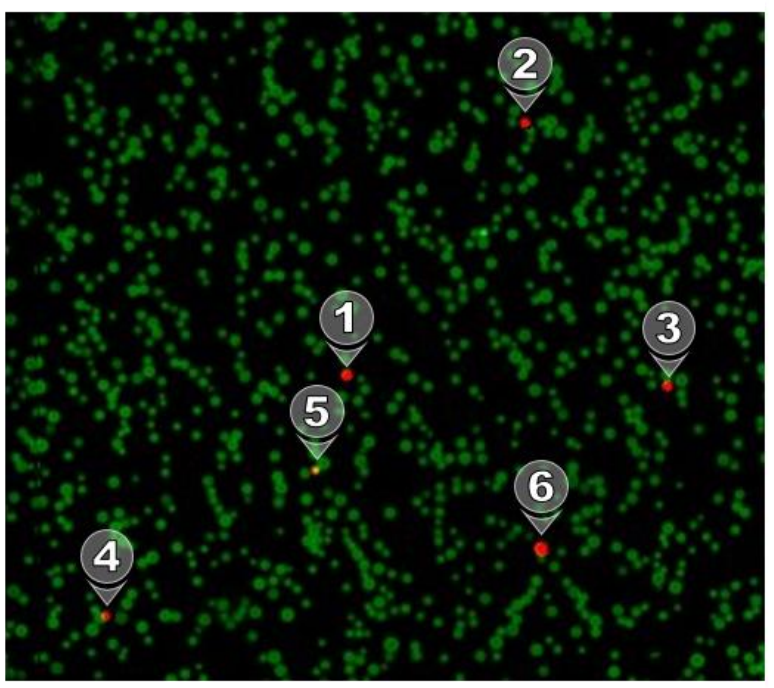

\section{C}

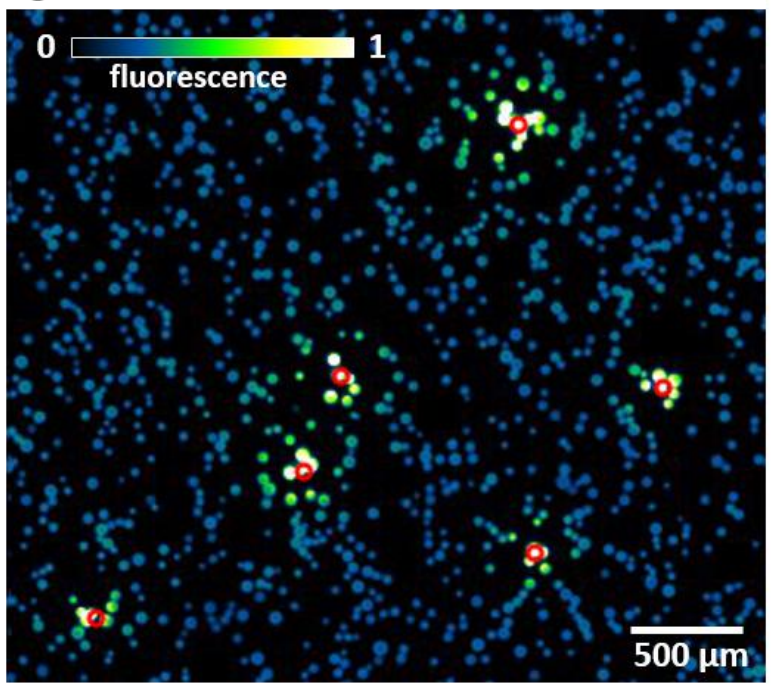

d

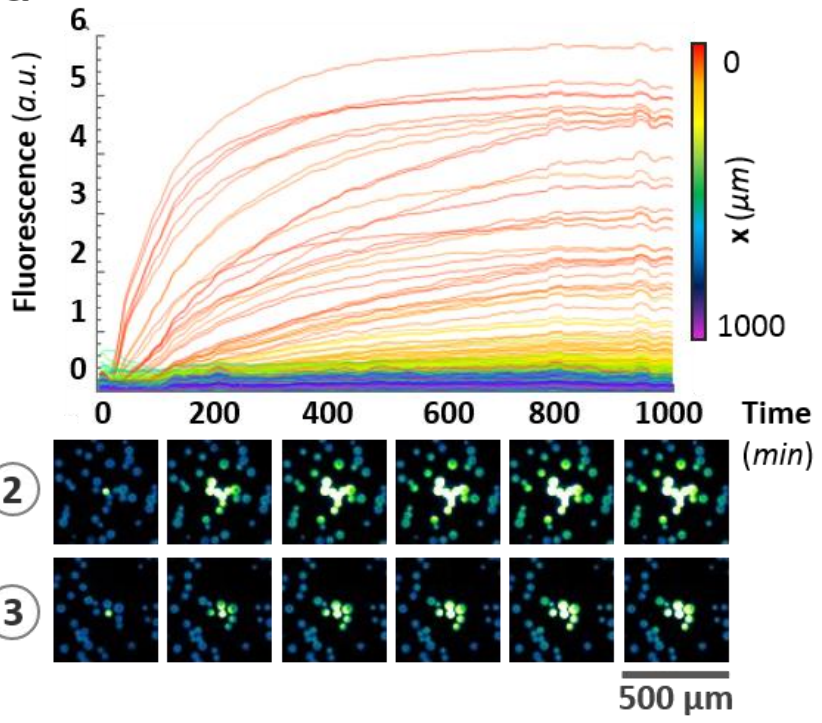

e
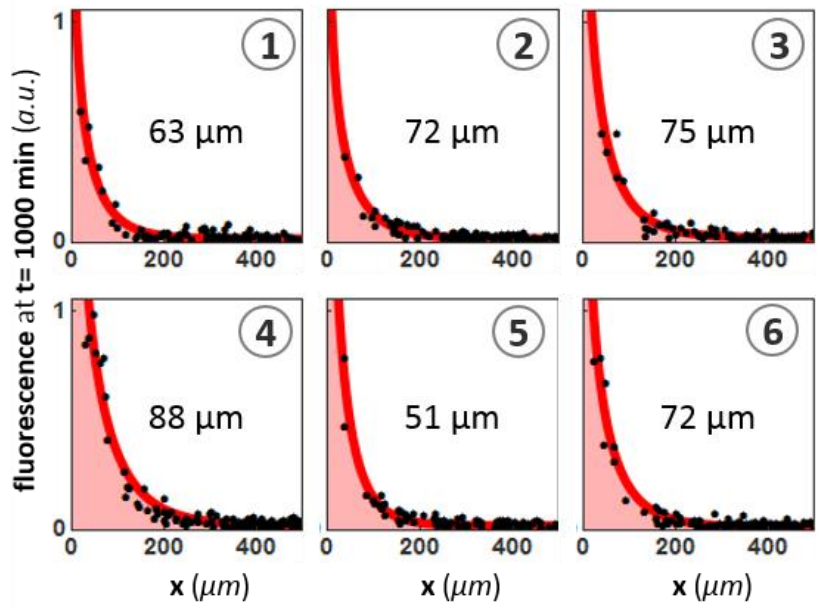

f
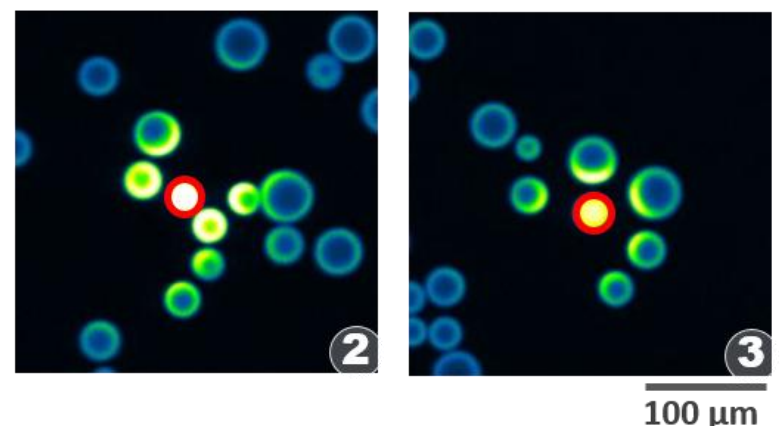

Figure 5. Mixed populations of symbiotic particles form localized clusters of activity around the minority agents. a, Schematic representation of the system. b, Barcode image of a portion of the fluidic chamber $\left(\right.$ green $=\mathrm{P}_{\gamma_{\rightarrow} \delta}$, red $=$ $\mathrm{P}_{\delta_{\rightarrow} \gamma}$ c, Snapshot image of EvaGreen fluorescence at $\mathrm{t}=1000 \mathrm{~min}$. The minority particles are indicated by red circles. $\mathbf{d}$, The fluorescence of each majority particle is plotted as a function of its distance to the nearest minority particle. The bottom images represent fluorescence snapshots of two colonies at the corresponding times. e, Profile of reactivity for each colony. The fluorescence of each majority particle is plotted as a function of its distance to the minority particle 
and fitted with a Bessel function of the second kind (length parameters $1_{d}$ are indicated in each frame). f, Confocal image (10X magnification) of two colonies showing the anisotropy of reactivity for the surrounding majority particles.

particles so that they light up as soon as the system has fetched the distance information (Fig. 4f): $P \alpha_{M}$ are modified with a molecular beacon-like probe, which fluoresces upon binding and polymerization of the returning $\beta$ strands (Fig. 4g). The full system now differentiates the sender particles $P \alpha_{M}$ with a timing that is precisely controlled by their position relative to the nearest converter particles $\mathrm{P}_{\alpha \rightarrow \beta}$ (Fig. 4h) ${ }^{15}$.

One may note that the return front is much slower than the first front passing through $\mathrm{P} \alpha_{\mathrm{B}}$. It is also twice as slow than the propagation through $\mathrm{P} \beta_{\mathrm{B}}$ initiated by $\mathrm{P} \beta_{\mathrm{M}}$ (Fig. S14), demonstrating that this is not due to the $P \beta_{B}$ propagation system itself being intrinsically slower. We therefore conclude that it is the presence of active $P \alpha_{\mathrm{B}}$ that slows down the propagation of the $\beta$ front. This observation suggests that competition for catalytic resources, such as nickase and/or polymerase, happens even if the two networks are spatially separated and occupy only a minor fraction of the total enzymatic mixture $^{27,28}$ (see also Fig. S10).

\section{Colony formation by symbiotic particles}

The experiments described above show that beads can behave as autonomous entities, but it is also possible to engineer synergic dependency and cooperativity among the individual agents. To evaluate this possibility, we split an autocatalytic loop into two templates (one encoding $\gamma$ to $\delta$ and one $\delta$ to $\gamma$ ), which are separately attached to different microspheres (Fig. 5a). An active PFL network now necessarily requires oligonucleotides to be exchanged between both bead types. We created flat fluidic chambers containing a high density of $\mathrm{P}_{\gamma_{\rightarrow} \delta}$ particles $\left(10^{5}\right.$ part./cm $\left.{ }^{2}\right)$ and a few $\mathrm{P}_{\delta_{\rightarrow \gamma}}$ particles (Fig. 5b). Real-time fluorescence monitoring revealed the emergence of localized activation colonies located around the minority particles (Fig. 5c and Supplementary Movie M6). These colonies emerge and grow to reach a steady state (Fig. 5d). The activity within the majority population is directly related to the presence of a minority bead in their neighborhood. The steady state fluorescence of the majority beads decays quickly with the distance to their feeding $\mathrm{P}_{\delta_{\rightarrow} \gamma}$, and fitting yield a characteristic length on the order of $50 \mu \mathrm{m}$ (Fig. 5e). Interestingly, when we zoom on established isolated colonies, we observe anisotropy in the majority particle's activity, the side facing the minority partner being more active than the other (Fig. 5f and Fig. S15). This confirms that the system dissipates enough free energy to maintain strong steady-state gradients over micrometric $(\sim 10 \mu \mathrm{m})$ distances (in the absence of an active sustaining mechanism such gradients would equilibrate in $\mathrm{r}^{2} / \mathrm{D} \approx 1 \mathrm{~s}$, see Supplementary Note 1).

In the experiment depicted in Figure 6, we gradually increased the density of minority particles from 20 to 2000 part. $/ \mathrm{cm}^{2}$. At low $\mathrm{P}_{\delta_{\rightarrow \gamma}}$ densities (20 to 300 part. $/ \mathrm{cm}^{2}$ ), the colonies are small, well defined, and similar for all conditions. However, when the density of minority beads reaches a threshold $\left(\sim 300\right.$ part. $\left./ \mathrm{cm}^{2}\right)$, we observe a sudden increase in the activity of most majority particles (jump in the fluorescence distribution, Fig. 6c). Since this happens when the mean distance between minority beads becomes close to the colonies characteristic length, it suggests that a colony-to-colony cooperative activation mechanism comes to play, where the signal strand diffusing from one colony is able to activate majority beads from a second colony, reinforcing it, and beneficiating in return. We therefore observe a two-level communication mechanism where $\mathrm{P}_{\gamma_{\rightarrow} \delta}$ beads locally detect the presence of a minority bead in their vicinity, but also globally sense the average density of the minority partners through a presumed percolation mechanism associated with a sharp density threshold. Toy-model simulations of this experiment reveal a similar behaviour, where the activity of the majority particles sharply increases when exceeding a threshold in the density of the minority particles (Fig. 6 b-c).

\section{Conclusion}

Communication using diffusive compounds is a general biological strategy to build multi-agents behaviours at microscopic scales. Diffusive cell signaling is typically classified into autocrine (self-communication) or paracrine (cross-communication)signaling ${ }^{29}$. These two types are naturally implemented in our system because the encoding templates can be designed to use either endogenous, exogenous or both signals as inputs. Our strategy to give memory and differentiated states to the agents involves autocrine bistable circuits based on positive feedback loops ${ }^{30,31}$. In the absence of physical boundaries, this approach requires that each particle is able to independently maintain its high state, i.e. compensate for the signals lost by diffusion. Analytically solving a source and decay model in the case of an isolated particle carrying a positive feedback loop exposes the experimental requirements (Supplementary Note 2): our results predict the existence of a critical size, or critical grafting density, below which a particle loses the ability to maintain a differentiated state. In other words, competition between global and local effects imposes that smaller agents dissipate faster in order to maintain their individuality. Below these values, only global behaviours can be observed. Downscaling the size of the particle would require programmable chemical 
systems with faster kinetics (for example, faster production rate) to withstand diffusion. Improving the enzymes, modifying the grafting chemistry or decreasing diffusion using crowding agents ${ }^{32}$ might be means to achieve such goal.

a experimental results
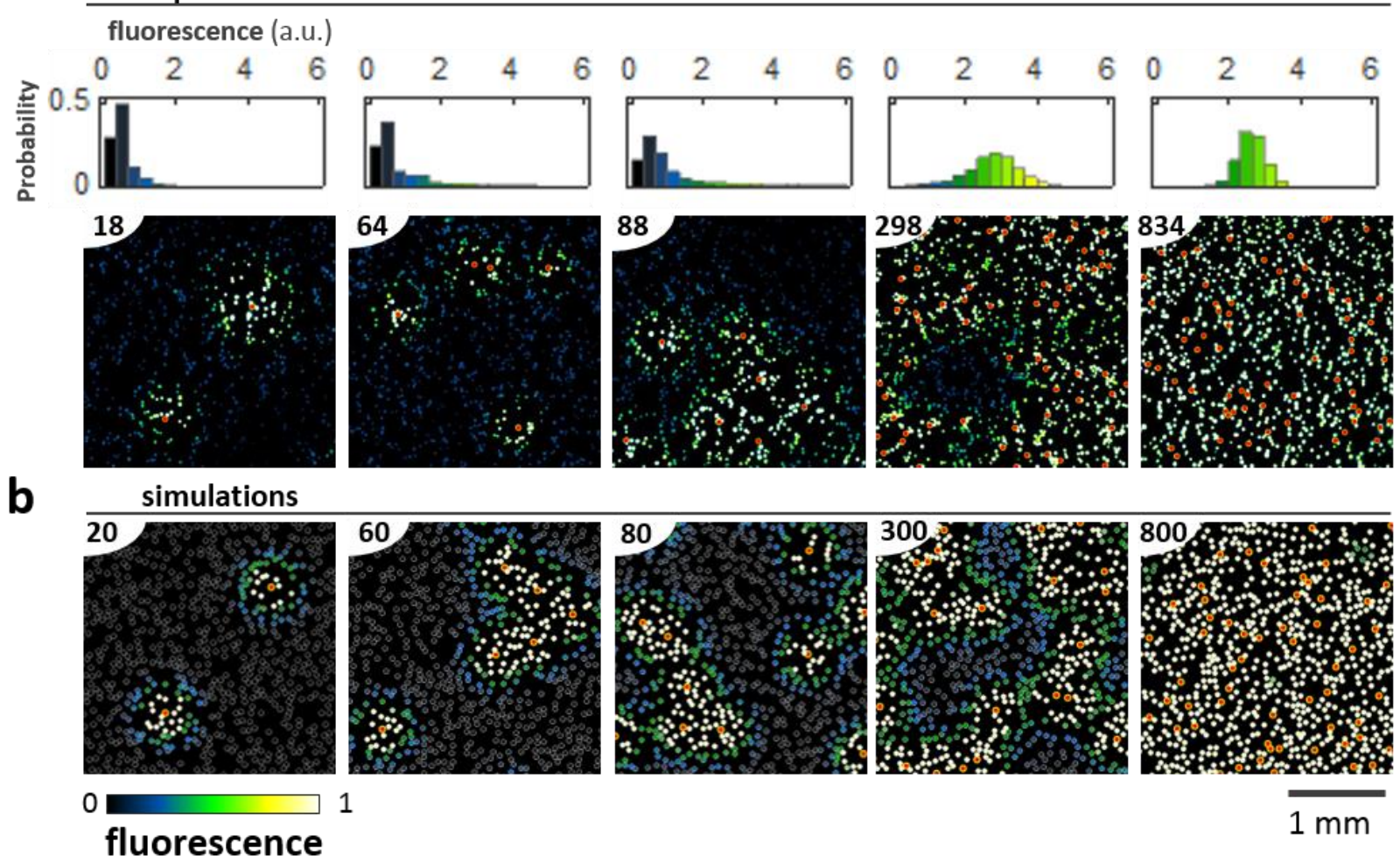

b
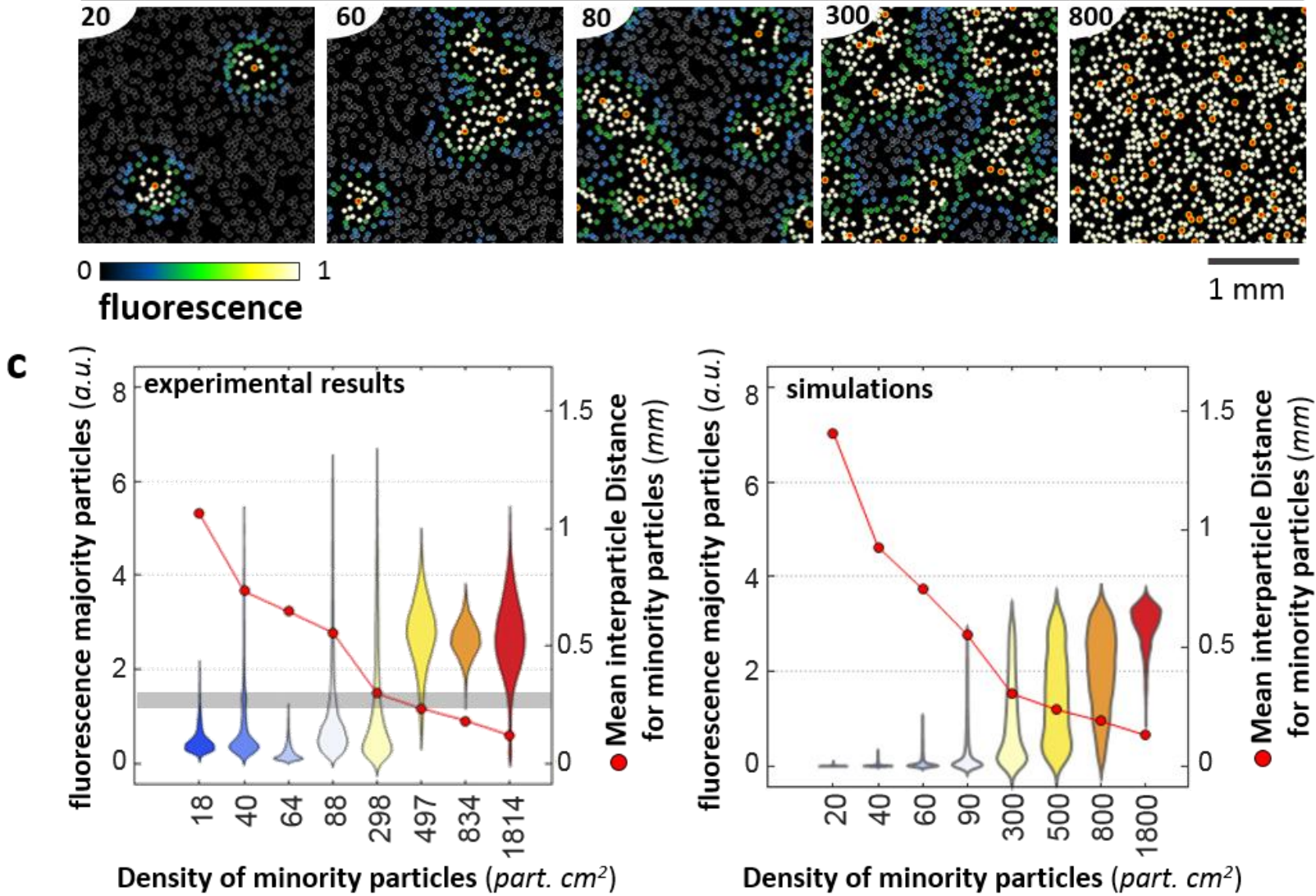

Figure 6. A cooperative mechanism reinforces symbiotic clusters. a, Experimental microscopy snapshots of colonies at the steady state $\left(\mathrm{t}=1000 \mathrm{~min}\right.$ ) for different densities (expressed in part./ $\mathrm{cm}^{2}$ in the corner of each snapshot) of minority particles $\mathrm{P}_{\delta_{\rightarrow \gamma}}$ (indicated by red circles). The top histograms represent the probability (Y-axis) distribution of the particles fluorescence (X-axis, expressed in a.u.). b. This behaviour is well reproduced by a model that numerically solves for the steady state activity of a random array of symbiotic particles. c, Experimental (left) and simulated (right) distributions of the fluorescence of the overall majority population $\left(\mathrm{P}_{\gamma_{\rightarrow} \delta}\right)$ for each density of minority particles. The threshold separating low and high levels of activity (gray stripe) corresponds to a mean distance between minority particles of $200-250 \mu \mathrm{m}$.

The competition between global and local effects has already been pointed out for other tethered DNA systems ${ }^{33}$ and imposes constraints on the miniaturization of molecular programming approaches. 
Compartmentalization techniques, such as microfabricated chambers, vesicles or emulsions ${ }^{34}$ provide an alternative to direct tethering of the DNA rules. Compartments efficiently restrict the diffusion of reactive species $^{35}$, but conversely require specific strategies in order to open communication channels between the units ${ }^{36-39}$. Living organisms elegantly manage transfer across their boundaries. Engineered bacteria have been used to implement collective behaviours, although the programming of living systems for such tasks remains challenging ${ }^{40-44}$.

Our strategy based on template-decorated particles provides the basic set of tools required to build and study artificial microscopic communities ${ }^{19}$. It uses a hierarchical approach that maintains a rational link from molecular pairwise interactions all the way to multiparticles dynamics. Compared to previous work in the study of collective behaviours ${ }^{45-47}$, the main advantage is a high level of programmability ${ }^{48}$ : one can freely decide the set of signals to which a given particle is sensitive, and its precise chemical behaviour in response to these signals. Our example with the "go-fetch" network has shown that, like in a multicellular organism, particles carrying different sets of templates can take on the various specialized roles necessary to achieve a global task. The colony experiment demonstrates fine sensing of the features of the local and global environment. While the particles used here are too large to display Brownian motion, one may envision that microscopic agents whose motility is controlled by the environment-sensitive network they carry could provide a path to dynamically self-shaping materials. In practical diagnostic applications, we also foresee that each particle could be programmed to independently perform a different sensing task, while collaborating with the others to provide a global consensus decision ${ }^{49}$.

\section{Methods}

Methods and associated references are available in the two Supplementary files.

\section{Acknowledgments}

This work was supported by the JSPS with a Grant-inAid from the JSPS for Scientific Research on Innovative Areas "Synthetic Biology for Comprehension of Biomolecular Networks" (\#23119001), and an ERC Consolidator grant "ProFF" (\#647275). G.G. acknowledges financial support by the JSPS Postdoc program. We thank Nicolas Bredeche, Nathanael AubertKato and Anthony Genot for advices and Yannick Tauran and Alexandre Baccouche for expressing and purifying the exonuclease. Correspondence should be sent to Y.R. (yannick.rondelez@espci.fr)

\section{Author contributions}

G.G. designed the study, performed experiments, analyzed the data and wrote the manuscript. A.Z. did mathematical analysis, contributed to image analysis and manuscript writing. J.C.G, A.E.T. contributed to experimental setup and designed the study. T.F. provided support with the microfluidic platform. Y.R. conceived, designed, and supervised the study, analyzed the data and wrote the manuscript.

\section{Additional information}

Supplementary information is available for this paper. Correspondence and requests for materials should be addressed to Y.R.

\section{References}

1. Padirac, A., Fujii, T. \& Rondelez, Y. Nucleic acids for the rational design of reaction circuits. Curr. Opin. Biotechnol. 24, 575-580 (2013).

2. Zhang, D. Y. \& Seelig, G. Dynamic DNA nanotechnology using strand-displacement reactions. Nat. Chem. 3, 103-113 (2011).

3. Han, D. et al. A cascade reaction network mimicking the basic functional steps of adaptive immune response. Nat. Chem. 7, 835-841 (2015).

4. Chen, Y.-J. et al. Programmable chemical controllers made from DNA. Nat. Nanotechnol. 8, 755-762 (2013).

5. Montagne, K., Plasson, R., Sakai, Y., Fujii, T. \& Rondelez, Y. Programming an in vitro DNA oscillator using a molecular networking strategy. Mol. Syst. Biol. 7, 466 (2011).

6. Kim, J. \& Winfree, E. Synthetic in vitro transcriptional oscillators. Mol. Syst. Biol. 7, 465 (2011).

7. Kim, J., White, K. S. \& Winfree, E. Construction of an in vitro bistable circuit from synthetic transcriptional switches. Mol. Syst. Biol. 2, 68 (2006).

8. Padirac, A., Fujii, T. \& Rondelez, Y. Bottom-up construction of in vitro switchable memories. Proc. Natl. Acad. Sci. 109, E3212-E3220 (2012).

9. Scalise, D. \& Schulman, R. Designing modular reactiondiffusion programs for complex pattern formation. TECHNOLOGY 02, 55-66 (2014).

10. Zadorin, A. S., Rondelez, Y., Galas, J.-C. \& Estevez-Torres, A. Synthesis of Programmable Reaction-Diffusion Fronts Using DNA Catalyzers. Phys. Rev. Lett. 114, 068301 (2015).

11. Padirac, A., Fujii, T., Estévez-Torres, A. \& Rondelez, Y. Spatial Waves in Synthetic Biochemical Networks. J. Am. Chem. Soc. 135, 14586-14592 (2013).

12. Zambrano, A., Zadorin, A. S., Rondelez, Y., Estevez-Torres, A. \& Galas, J.-C. Pursuit-and-evasion Reaction-diffusion Waves In Micro-reactors with Tailored Geometry. J. Phys. Chem. B (2015). doi:10.1021/jp509474w

13. Miller, M. B. \& Bassler, B. L. Quorum Sensing in Bacteria. Annu. Rev. Microbiol. 55, 165-199 (2001).

14. Waters, C. M. \& Bassler, B. L. QUORUM SENSING: Cell-toCell Communication in Bacteria. Annu. Rev. Cell Dev. Biol. 21, 319-346 (2005).

15. Pourquié, O. The Segmentation Clock: Converting Embryonic Time into Spatial Pattern. Science 301, 328-330 (2003).

16. Baker, R. E., Schnell, S. \& Maini, P. K. A clock and wavefront mechanism for somite formation. Dev. Biol. 293, 116-126 (2006). 
17. Kessin, R. H. Dictyostelium: Evolution, Cell Biology, and the Development of Multicellularity. (Cambridge University Press, 2001).

18. Baccouche, A., Montagne, K., Padirac, A., Fujii, T. \& Rondelez, Y. Dynamic DNA-toolbox reaction circuits: A walkthrough. Methods 67, 234-249 (2014).

19. Yashin, V. V., Kolmakov, G. V., Shum, H. \& Balazs, A. C. Designing Synthetic Microcapsules that Undergo Biomimetic Communication and Autonomous Motion. Langmuir (2015). doi:10.1021/acs.langmuir.5b01862

20. Taylor, A. F., Tinsley, M. R. \& Showalter, K. Insights into collective cell behaviour from populations of coupled chemical oscillators. Phys. Chem. Chem. Phys. (2015).

21. Yashin, R., Rudchenko, S. \& Stojanovic, M. N. Networking Particles over Distance Using Oligonucleotide-Based Devices. J. Am. Chem. Soc. 129, 15581-15584 (2007).

22. Jung, C., Allen, P. B. \& Ellington, A. D. A stochastic DNA walker that traverses a microparticle surface. Nat. Nanotechnol. 11, 157-163 (2016).

23. Soh, S., Byrska, M., Kandere-Grzybowska, K. \& Grzybowski, B. A. Reaction -Diffusion Systems in Intracellular Molecular Transport and Control. Angew. Chem. Int. Ed Engl. 49, 4170-4198 (2010).

24. Tan, E. et al. Specific versus Nonspecific Isothermal DNA Amplification through Thermophilic Polymerase and Nicking Enzyme Activitiest. Biochemistry (Mosc.) 47, 9987-9999 (2008).

25. Montagne, Kevin, Gines, Guillaume, Fujii, Teruo \& Rondelez, Yannick. Creative destruction: Boosting functionality of synthetic DNA circuits with tailored degradation. Rev.

26. Davis, R. M., Muller, R. Y. \& Haynes, K. A. Can the Natural Diversity of Quorum-Sensing Advance Synthetic Biology? Front. Bioeng. Biotechnol. 3, (2015).

27. Genot, A. J., Fujii, T. \& Rondelez, Y. Scaling down DNA circuits with competitive neural networks. J. R. Soc. Interface 10, 20130212 (2013).

28. Rondelez, Y. Competition for Catalytic Resources Alters Biological Network Dynamics. Phys. Rev. Lett. 108, 018102 (2012)

29. Youk, H. \& Lim, W. A. Secreting and Sensing the Same Molecule Allows Cells to Achieve Versatile Social Behaviours. Science 343, 1242782 (2014).

30. Brandman, O. \& Meyer, T. Feedback Loops Shape Cellular Signals in Space and Time. Science 322, 390-395 (2008).

31. Wolf, D. M. \& Arkin, A. P. Motifs, modules and games in bacteria. Curr. Opin. Microbiol. 6, 125-134 (2003).

32. Dauty, E. \& Verkman, A. S. Molecular crowding reduces to a similar extent the diffusion of small solutes and macromolecules: measurement by fluorescence correlation spectroscopy. J. Mol. Recognit. 17, 441-447 (2004).

33. Teichmann, M., Kopperger, E. \& Simmel, F. C. Robustness of Localized DNA Strand Displacement Cascades. ACS Nano (2014). doi:10.1021/nn503073p
34. Hasatani, K. et al. High-throughput and long-term observation of compartmentalized biochemical oscillators. Chem. Commun. Camb. Engl. 49, 8090-8092 (2013).

35. Toiya, M., González-Ochoa, H. O., Vanag, V. K., Fraden, S. \& Epstein, I. R. Synchronization of Chemical Microoscillators. J. Phys. Chem. Lett. 1, 1241-1246 (2010).

36. Tayar, A. M., Karzbrun, E., Noireaux, V. \& Bar-Ziv, R. H. Propagating gene expression fronts in a one-dimensional coupled system of artificial cells. Nat. Phys. 11, 1037-1041 (2015).

37. Dewey, D. C., Strulson, C. A., Cacace, D. N., Bevilacqua, P. C. \& Keating, C. D. Bioreactor droplets from liposomestabilized all-aqueous emulsions. Nat. Commun. 5, 4670 (2014).

38. Villar, G., Graham, A. D. \& Bayley, H. A Tissue-Like Printed Material. Science 340, 48-52 (2013).

39. Weitz, M. et al. Communication and Computation by Bacteria Compartmentalized within Microemulsion Droplets. J. Am. Chem. Soc. 136, 72-75 (2014).

40. Lou, C. et al. Synthesizing a novel genetic sequential logic circuit: a push-on push-off switch. Mol. Syst. Biol. 6, 350 (2010).

41. Elowitz, M. B. \& Leibler, S. A synthetic oscillatory network of transcriptional regulators. Nature 403, 335-338 (2000).

42. Danino, T., Mondragón-Palomino, O., Tsimring, L. \& Hasty, J. A synchronized quorum of genetic clocks. Nature 463, 326-330 (2010).

43. Amemiya, T. et al. Collective and individual glycolytic oscillations in yeast cells encapsulated in alginate microparticles. Chaos Interdiscip. J. Nonlinear Sci. 25, 064606 (2015).

44. Basu, S., Gerchman, Y., Collins, C. H., Arnold, F. H. \& Weiss, $R$. A synthetic multicellular system for programmed pattern formation. Nature 434, 1130-1134 (2005).

45. Tinsley, M. R., Taylor, A. F., Huang, Z. \& Showalter, K. Emergence of Collective Behaviour in Groups of Excitable Catalyst-Loaded Particles: Spatiotemporal Dynamical Quorum Sensing. Phys. Rev. Lett. 102, 158301 (2009).

46. Taylor, A. F., Tinsley, M. R., Wang, F., Huang, Z. \& Showalter, K. Dynamical Quorum Sensing and Synchronization in Large Populations of Chemical Oscillators. Science 323, 614-617 (2009).

47. Golestanian, R. Collective Behaviour of Thermally Active Colloids. Phys. Rev. Lett. 108, 038303 (2012).

48. Aubert, N., Mosca, C., Fujii, T., Hagiya, M. \& Rondelez, Y. Computer-assisted design for scaling up systems based on DNA reaction networks. J. R. Soc. Interface 11, 20131167 (2014).

49. Binder, S. R., Hixson, C. \& Glossenger, J. Protein arrays and pattern recognition: New tools to assist in the identification and management of autoimmune disease. Autoimmun. Rev. 5, 234-241 (2006). 